\title{
Immunogenicity evaluation of MS2 phage-mediated chimeric nanoparticle displaying an immunodominant B cell epitope of foot-and-mouth disease virus
}

\author{
Guoqiang Wang ${ }^{1,2}$, Yunchao Liu ${ }^{2}$, Hua Feng ${ }^{2}$, Yumei Chen ${ }^{3}$, Suzhen Yang ${ }^{2}$, Qiang Wei ${ }^{2}$, Juan Wang ${ }^{4}$, \\ Dongmin Liu ${ }^{4}$, Gaiping Zhang ${ }^{\text {Corresp. } 1,2,5}$ \\ ${ }^{1}$ College of Animal Science and Veterinary Medicine, Henan Agricultural University, Zhengzhou, China \\ 2 \\ 3 School of Life Sciences, Zhengzhou University, Zhengzhou, China \\ 4 Henan Zhongze Biological Engineering Co., Ltd, Zhengzhou, China \\ 5 Jiangsu Co-Innovation Center for the Prevention and Control of Important Animal Infectious Disease and Zoonose, Yangzhou University, Yangzhou, China \\ Corresponding Author: Gaiping Zhang \\ Email address: zhanggaiping2003@163.com
}

Foot-and-mouth disease (FMD) is a highly contagious disease of cloven-hoofed animals that has caused tremendous economic losses worldwide. In this study, we designed a chimeric nanoparticles (CNPs) vaccine that displays the predominant epitope of the serotype $\mathrm{O}$ foot-and-mouth disease virus (FMDV) VP1 131-160 on the surface of MS2 phage. The recombinant protein was expressed in Escherichia Coli and can self-assemble into CNPs with diameter at $25-30 \mathrm{~nm}$ in vitro. A tandem repeat peptide epitopes (TRE) was prepared as control. Mice were immunized with CNPs, TRE and commercialized synthetic peptide vaccines (PepVac), respectively. The ELISA results showed that CNPs stimulated a little higher specific antibody levels to PepVac, but was significantly higher than TRE groups. Moreover, the results from specific IFN- $\gamma$ responses and lymphocyte proliferation test indicated that CNPs immunized mice exhibited significantly enhanced cellular immune response compared to TRE. These results suggested that the CNPs constructed in current study could be a potential alternative vaccine in future FMDV control. 
1

4 Guoqiang Wang ${ }^{1,2}$, Yunchao Liu ${ }^{2}$, Hua Feng ${ }^{2}$, Yumei Chen ${ }^{3}$, Suzhen Yang ${ }^{2}$, Qiang wei ${ }^{2}$, Juan

5 Wang ${ }^{4}$, Dongmin Liu ${ }^{4}$, Gaiping Zhang ${ }^{1,2,5, *}$

6

$7 \quad$ College of Animal Science and Veterinary Medicine, Henan Agricultural University, Zhengzhou,

8 450002, China

$9 \quad{ }^{2}$ Henan Academy of Agricultural Sciences, Henan Provincial Key Laboratory of Animal

\section{Immunogenicity evaluation of MS2 phage-mediated chimeric nanoparticle displaying an immunodominant $B$ cell epitope of foot-and-mouth disease virus}

Immunology, Zhengzhou, 450002, China

${ }^{3}$ School of Life Sciences, Zhengzhou University, Zhengzhou, 450001, China

${ }^{4}$ Henan Zhongze Biological Engineering Co., Ltd., Zhengzhou, 450019, China.

5 Jiangsu Co-Innovation Center for the Prevention and Control of Important Animal Infectious

Disease and Zoonose, Yangzhou University, Yangzhou 225009, PR China

16

7

* Gaiping Zhang, Corresponding author, E-mail: zhanggaiping2003@163.com

Tel: +86037165723268, Fax: +86037165738179 


\section{ABSTRACT}

Foot-and-mouth disease (FMD) is a highly contagious disease of cloven-hoofed animals that has caused tremendous economic losses worldwide. In this study, we designed a chimeric nanoparticles (CNPs) vaccine that displays the predominant epitope of the serotype $\mathrm{O}$ foot-andmouth disease virus (FMDV) VP1 131-160 on the surface of MS2 phage. The recombinant protein was expressed in Escherichia Coli and can self-assemble into CNPs with diameter at 25$30 \mathrm{~nm}$ in vitro. A tandem repeat peptide epitopes (TRE) was prepared as control. Mice were immunized with CNPs, TRE and commercialized synthetic peptide vaccines (PepVac), respectively. The ELISA results showed that CNPs stimulated a little higher specific antibody levels to PepVac, but was significantly higher than TRE groups. Moreover, the results from specific IFN- $\gamma$ responses and lymphocyte proliferation test indicated that CNPs immunized mice exhibited significantly enhanced cellular immune response compared to TRE. These results suggested that the CNPs constructed in current study could be a potential alternative vaccine in future FMDV control.

\section{INTRODUCTION}

Foot-and-mouth disease virus (FMDV) infects over 70 species of cloven-hoofed animals and has caused enormous economic losses to stockbreeding industry worldwide (Diaz-San Segundo et al. 2016; Pereira 1976). Conventional inactivated FMDV vaccine has been widely used and played a crucial role in epidemic control and eradication of FMDV globally. However, it remains some problems of the inactivated FMDV vaccine, such as the risk of virus escape during vaccine production and difficulty in differentiating infected from vaccinated animals, which limit the application of inactivated FMDV vaccine in emergency control programs (Dong et al. 2015; Parida 2009; Wang et al. 2002). Epitope-based polypeptide vaccines are well known for their superior abilities to provide more effective antigens and it could be effectively distinguished between infected and vaccinated animals. However, limited cellular immune response and immune protection were induced by these epitope vaccines in large host animals, resulting in the failure of FMDV prevention (Rodriguez et al. 2003; Taboga et al. 1997).

Neutralizing epitopes of FMDV, which distributed in structural proteins VP1, VP2 and VP3, is critical to an effective immune protection. FMDV VP1 G-H loop has been identified as a primary antigenic epitope, which can effectively induce FMDV specific neutralizing antibodies 
53

54

55

56

57

58

59

60

61

62

63

64

(Bittle et al. 1982; DiMarchi et al. 1986). The highly conserved arginine-glycine-aspartic acid (RGD) motif is located on the top of G-H loop of VP1. FMDV can use RGD-binding integrins as receptors to initiate infection (Burman et al. 2006). Therefore, based on the dominant epitope G$\mathrm{H}$ loop, a large number of FMD epitope vaccines are being studied. A commercial FMD synthetic-peptide vaccine (UBITh ${ }^{\circledR}$ vaccine) containing an entire G-H loop domain and extensive flanking sequences (129-169) was licensed for use in china(Cao et al. 2016).

Nanoparticles-based antigen display technology provides an approach to improve the immune response and immune protection effect of subunit vaccines (Chackerian 2007; Crisci et al. 2009; Crisci et al. 2012; Xu et al. 2017). MS2 phage is a novel display and delivery platform for foreign peptide epitopes. MS2 phage belongs to the Leviviridae family with small positivesense single-stranded RNA bacteriophages, which is encapsulated by an icosahedral capsid comprised of 180 copies of coat protein(CP) and a single copy of mature protein (AP) (Koning et al. 2016; Wei et al. 2008). The CP of MS2 phage can self-assemble to form virus-like particles (VLPs) in Escherichia Coli (E. coli) in the absence of viral RNA (Caldeira \& Peabody 2011; Lino et al. 2017). The AB loop of CP is exposed on the surface of the phage particles, which tolerates exogenous peptides insertion without affecting the self-assembly of CP (Fu \& Li 2016; Mastico et al. 1993). These characteristics play an important fundamental basis for remodeled epitope peptide to enhance the efficacy of vaccines.

In the present research, we inserted the epitope sequence (131-160, including G-H loop and its flanking sequence) into the $\mathrm{AB}$ loop of MS2 phage CP. Chimeric protein was expressed in $E$. coli and self-assembled into chimeric nanoparticles (CNPs). The immunoassay test showed CNPs could efficiently enhance the antibody levels and cellular immune response compared to tandem repeat peptide epitopes (TRE) and commercialized synthetic peptide vaccine (PepVac) groups. Together, our results suggested that MS2- mediated CNPs provide a favorable platform for displaying foreign epitopes and an innovative approach to develop alternative vaccines for FMDV.

\section{MATERIALS AND METHODS}

\section{Animal, commercial vaccine and antibody}

Twenty six-week-old female Kunming mice were provided by Laboratory Animal Center, Zhengzhou University. This study was performed with the approval of the Animal Experiment Committee of Henan Academy of Agricultural Sciences (Approval number SYXK 2014-0007). 
84

All animals used in this study were humanely maintained and euthanized according to the animal ethics procedures and guidelines of China.

Commercialized synthetic peptide vaccine (peptide 2600+2700+2800) (PepVac) was purchased from Shang hai Shen-lian Biotmedical Corporation (http://www.shenlianbiotech.com.cn/product-4.html). 2600, 2700 and 2800 represent the epitope sequences of pandemic strains of ME-SA topotypes, CATHAY topotypes and SEA topotypes of serotype O FMDV, respectively.

The monoclonal antibodies against FMDV VP1 G-H loop (141-160) was prepared and provided by the Key Laboratory of Animal Immunology, Henan Academy of Agricultural Sciences, which was generated using hybridoma technology as described in previous reference(Ma et al. 2010). Binary ethylenimine (BEI) inactivated FMDV, guinea pigs antiFMDV/O hyper-immune serum and rabbit polyclonal antibodies against FMDV were obtained from the National FMD Reference Laboratory of the People's Republic of China. Horseradish peroxidase (HRP)-conjugated goat anti guinea pigs IgG was obtained from Sigma, and Horseradish peroxidase (HRP)-conjugated goat anti mouse IgG was obtained from Abcam.

\section{Plasmid construction}

All the primers used to construct the recombinant plasmids were shown in Table 1. The restriction sites BamH I and Hind III were represented in lowercase in primers MS2-F and MS2$\mathrm{R}$. The sequences in bold type in two primers IN-F and IN-R were the epitope sequence 131-160 of VP1, and underlined 36 nucleotides were reverse complementary. The epitope sequence 131160 came from the O/BY/CHA/2010 strain (GenBank: AET43040.1). The cDNA of encoding AP and CP sequences of MS2 were reversely transcribed from MS2 genome mRNAs (purchased from Sigma) by primers MS2-F and MS2-R using a One-Step RT-PCR Kit (TaKaRa) and cloned into T-Vector $\mathrm{pMD}^{\mathrm{TM}} 19$ (Simple). The recombinant plasmid was named as T-MS2. The construction diagrams of recombinant plasmids were depicted in Fig.1. Fragment including AP and $\mathrm{CP}$ sequences was amplified from T-MS2 plasmid using primers MS2-F and MS2-R and cloned into pET28a plasmid named as pCP. The whole chimeric sequence was amplified by overlap extension PCR (OE-PCR) (Liu et al. 2006; Yang et al. 2005). Briefly, the upstream and downstream fragments were amplified from T-MS2 plasmid using MS2-F/IN-R and MS2-R/INF primer pairs, respectively. The mixture of equimolar upstream fragment and downstream fragment was used as template, and then the whole chimeric sequences containing $\mathrm{AP}$ gene, $\mathrm{CP}$ 
115

116

117

118

119

120

121

122

123

gene and the epitope sequence 131-160 were amplified using primers MS2-F and MS2-R and

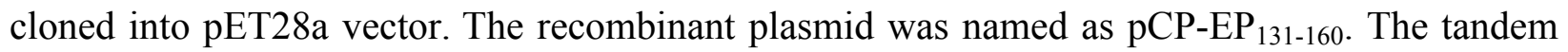
repeat sequence consisting of 3 copies of the epitope sequence 131-160 connected by GSGSGS was artificially synthesized and cloned into pET28a vector named as p-(EP $\left.\mathrm{EP}_{131-160}\right)_{3}$. Recombinant plasmids pCP and pCP-EP ${ }_{131-160}$ were identified by digestion with restriction enzymes BamH I and Hind III, respectively, and p-( $\left.\mathrm{EP}_{131-160}\right)_{3}$ was identified by restriction enzymes Nde I and Xol I. Finally, all the recombinant plasmids were identified by sequencing.

\section{Recombinant protein expression and purification}

Recombinant plasmids pCP, pCP-EP $\mathrm{E}_{131-160}$ and p-( $\left.\mathrm{EP}_{131-160}\right)_{3}$ were transformed into E. Coli BL21 (DE3), respectively. A single clone was selected from LB agar plate and cultured in LB medium supplemented with $50 \mu \mathrm{g} / \mathrm{ml}$ kanamycin. Until the $\mathrm{OD}_{600}$ reached 0.8 , target proteins were induced by $0.3 \mathrm{mM}$ isopropyl $\beta$-D-thiogalactoside (IPTG). After induction at $20^{\circ} \mathrm{C}$ for $16 \mathrm{~h}$, the cells were harvested by centrifugation at $6,000 \times \mathrm{g}$ for $15 \mathrm{~min}$ at $4{ }^{\circ} \mathrm{C}$. The cell pellet was resuspended in PBS buffer and sonicated seven times for $30 \mathrm{~s}$ each on ice, and then was centrifuged at $12,000 \times \mathrm{g}$ for $15 \mathrm{~min}$ at $4{ }^{\circ} \mathrm{C}$. The soluble fraction in supernatant and insoluble fraction in precipitation were analyzed using SDS-PAGE and Western blotting. The soluble CP and chimeric protein in supernatant were purified separately as below: DNase I and RNase A with a final concentration of $1 \mu \mathrm{g} / \mathrm{ml}$ were added into the supernatant at room temperature for 30 min, respectively. Then solid $\mathrm{NaCl}$ with a final concentration $1 \mathrm{~mol} / \mathrm{L}$ was added and incubated on ice for $1 \mathrm{~h}$. After centrifugation at $11,000 \times \mathrm{g}$ for $10 \mathrm{~min}$, PEG8000 was added into supernatant to a final concentration of $10 \%(\mathrm{w} / \mathrm{v})$ and stored the mixture for at least $1 \mathrm{~h}$. After centrifuged again, the pellet was re-suspended in PBS buffer. Followed by incubated with an equal volume of chloroform and then gently vortexed the mixture for 30 seconds, finally the aqueous phases containing chimeric protein were collected by centrifuged at 5,000× $\mathrm{g}$ for $10 \mathrm{~min}$. The chimeric protein was further purified by gel filtration chromatography (Capto Core 700, GE). Briefly, preliminary purified chimeric protein was pumped onto PBS buffer equilibrated Capto Core 700 column and the effluent containing target protein was collected directly.

Besides, the tandem repeat peptide epitopes (TRE) was purified by Ni-NTA column (Merck, Germany). TRE was expressed as inclusion body (IB), so IB was dissolved in $8 \mathrm{M}$ urea and loaded onto Ni-NTA column equilibrated with $0.05 \mathrm{M}$ carbonate buffer $(\mathrm{pH}=9.0)$ containing $8 \mathrm{M}$ urea. After washing with 10 beds of carbonate buffer (50 mM imidazole, $8 \mathrm{M}$ urea), the IB was 
146

147

148

149

150

151

152

153

154

155

156

157

158

159

160

161

162

163

164

165

166

167

168

169

170

171

172

173

174

175

eluted with carbonate buffer (100 $\mathrm{mM}$ imidazole, $8 \mathrm{M}$ urea). Then the purified protein was gradient dialyzed with $0.05 \mathrm{M}$ carbonate buffer $(\mathrm{pH}=9.0)$ containing continuously decreased urea concentration (from 8 to $0 \mathrm{M}$ ) for $72 \mathrm{~h}$. The concentration of purified recombinant proteins was calculated using Micro $\mathrm{BCA}^{\mathrm{TM}}$ protein assay kit (Thermo Scientific, USA) following the manufacturer's protocol.

\section{Identification of recombinant proteins}

The purified chimeric protein was further characterized by transmission electronic microscopy (TEM) using the negative staining and particle size was analyzed by dynamic light scattering (DLS) as described before (Chandramouli et al. 2013).

The reactivity of purified recombinant proteins was analyzed by Dot-ELISA. Purified CP, CNPs and TRE were blotted onto a nitrocellulose (NC) membrane. Inactivated FMDV was used as positive control. The NC membrane was blocked with 5\% skimmed milk for $2 \mathrm{~h}$ at $37^{\circ} \mathrm{C}$, and then incubated with guinea pigs anti-FMDV/O hyper-immune serum or anti-FMDV VP1 G-H loop (141-160) monoclonal antibodies as the primary antibodies, followed by a HRP-conjugated goat anti guinea pigs IgG or mouse IgG as secondary antibody. The Dot-ELISA was visualized using 3-amino-9-ethylcarbazole (AEC) (ZSGB-BIO, Beijing, China), which is a substrate of peroxidase.

\section{Vaccine preparation and immunization}

The purified CNPs and TRE were emulsified with adjuvant Montanide ISA 50V2 (Seppic, France) for animal vaccination, respectively. The ratio of aqueous antigen to the oil adjuvant was 1:1 (V/V). Twenty female Kunming mice were randomly divided into four groups (five each group). Groups 1 and 2 were vaccinated subcutaneously with $15 \mu \mathrm{g}$ of CNPs and $30 \mu \mathrm{g}$ of TRE, respectively. Group 3 was inoculated with $100 \mu \mathrm{l}$ of PepVac (more than $7.5 \mu \mathrm{g}$ ) used as positive control, and Group 4 was vaccinated of $100 \mu$ l of PBS with the same volume of adjuvant as negative control. All mice received boost vaccination at 28 days after first immunization.

\section{Detection of Anti-FMDV-Specific Antibodies}

Serum samples were collected weekly from the tail vein after the first immunization until the eighth week. All the samples were tested for anti-FMDV specific antibodies by ELISA. Briefly, $100 \mu \mathrm{l}$ per well rabbit polyclonal antibody against FMDV was coated on 96-well ELISA plate with and incubated at $4{ }^{\circ} \mathrm{C}$ overnight. The 96 -well plate was blocked with $250 \mu \mathrm{PBST}$ including 
176

177

178

179

180

181

182

183

184

185

186

187

188

189

190

191

192

193

194

195

196

197

198

199

200

201

202

203

204

205

$5 \%$ skimmed milk at $37{ }^{\circ} \mathrm{C}$ for $2 \mathrm{~h}$, then washed three times with PBST. 20-fold diluted inactivated FMDV was added to the 96 plate with $100 \mu \mathrm{l}$ per well and incubated at $37{ }^{\circ} \mathrm{C}$ for $1 \mathrm{~h}$. After washed with PBST, the serum samples with a dilution of 1:100 were added and incubated at room temperature for $1 \mathrm{~h}$. Then the plate was reacted with HRP-conjugated goat anti mouse IgG at $37^{\circ} \mathrm{C}$ for $1 \mathrm{~h}$. Finally, after washed five times, the reaction substrate was respectively added to each well and incubated at $37^{\circ} \mathrm{C}$ for 10 minutes. Then, the reaction was stopped by $2 \mathrm{M}$ $\mathrm{H}_{2} \mathrm{SO}_{4}$, and the $\mathrm{OD}_{450} \mathrm{~nm}$ values were measured by a spectrophotometer.

\section{Spleen lymphocyte proliferation assay}

The spleen lymphocytes were isolated from immunized mice at 28 days after booster immunization by using a lymphocyte separation kit (Solarbio, Beijing, China). The Cell Counting Kit-8 (CCK-8) (Solarbio, Beijing, China) was used to detect cell proliferation according to manufacture instruction. (2-(2-Methoxy-4-nitrophenyl)-3- (4-nitrophenyl)- 5-(2,4 -disulfobenzene)-2H-tetrazole monosodium salt) (WST-8) is the main component of the CCK-8 kit. Briefly, The spleen lymphocytes were re-suspended in RPMI-1640 medium containing 10\% FBS, and incubated in triplicate in 96-well plate with a density of $5 \times 10^{5}$ cells/well at $37^{\circ} \mathrm{C}$ for 24 h. Then, the cells were stimulated with $50 \mu \mathrm{l}$ of inactivated FMDV $(20 \mu \mathrm{g} / \mathrm{ml})$. Concanavalin A $(\mathrm{ConA}, 5 \mu \mathrm{g} / \mathrm{ml})$ and unstimulated wells were used as the positive control and negative control. After incubation at $37^{\circ} \mathrm{C}$ for $48 \mathrm{~h}$, WST-8 (10 $\mu \mathrm{l} /$ well) was added to each well and incubated at $37^{\circ} \mathrm{C}$ for $1 \mathrm{~h}$. The absorbance of each well was measured at $450 \mathrm{~nm}$. T lymphocyte proliferation was expressed as the stimulation index (SI), which was the ratio of $\mathrm{OD}_{450} \mathrm{~nm}$ of stimulated wells to $\mathrm{OD}_{450} \mathrm{~nm}$ of unstimulated ones.

\section{Cytokines detection}

Splenic lymphocytes culture supernatants stimulated with inactivated FMDV for $48 \mathrm{~h}$ were collected for evaluating the concentration of IL-2, IL-4 and IFN- $\gamma$. The assay and data calculation were performed by the commercially available ELISA kit (Bogoo, Shanghai, China) following manufacturers' instructions.

\section{Statistical analysis}

Statistical analysis was performed by using GraphPad Prism 7.0 software. Two-way ANOVA and t-test method were employed for significant test. Dates were shown as the mean \pm SEM, and P-values less than 0.05 was considered as statistically significant. 
206

207

208

209

210

211

212

213

214

215

216

217

218

219

220

221

222

223

224

225

226

227

228

229

230

231

232

233

234

\section{RESULTS}

\section{Construction of recombinant vectors}

A schematic diagram of obtaining the target gene and constructing three recombinant plasmids is outlined in Fig.1. The MS2 (AP+CP) gene, upstream fragment, downstream fragment and the whole length chimeric genes were amplified by PCR/OE-PCR with the expected molecular size of 1644 bp, 1260 bp, 487 bp and 1734 bp (Fig.2A), suggesting that the epitope 131-160 gene was successfully inserted into CP of MS2. Recombinant plasmids, pCP, pCP-EP ${ }_{131-160}$ and p-(EP $\left.\mathrm{EP}_{131-160}\right)_{3}$, were also confirmed by restriction digestion (Fig.2B) and sequencing (Shown in the supplemental information). The fragment sizes digested from

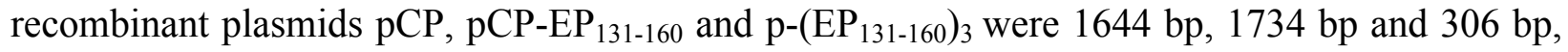
respectively, which were consistent with expected.

\section{Expression and purification of recombinant proteins}

To further investigate whether the expressed recombinant proteins are soluble in solution, SDS-PAGE and Western blotting were performed in our studies. The result of SDS-PAGE indicated that approximately $50 \%$ of $\mathrm{CP}(16 \mathrm{kDa})$ and chimeric proteins $(18 \mathrm{kDa})$ were expressed in soluble form, but TRE (10 kDa) was expressed as IB (Fig.3A). Additionally, the recombinant proteins of the chimeric protein and TRE could be recognized by the anti-FMDV VP1 G-H loop (141-160) monoclonal antibodies, while CP did not show the similar phenomenon (Fig.3B).The purities of three recombinant proteins were estimated to be over $85 \%$ (Fig.3C).

\section{Reactivity of CNPs and TRE}

The reactivity of recombinant proteins was analyzed by Dot-ELISA. The results showed that CNPs and TRE could efficiently react with anti-FMDV hyper-immune serum (Fig.4A) and antiFMDV VP1 G-H loop (141-160) monoclonal antibodies (Fig.4B), while CP did not show the similar phenomenon. These results suggested that the epitope sequence 131-160 was correctly displayed on the surface of A-B loop of MS2, and CNPs and TRE had a superior immune reactivity with anti-FMDV hyper-immune serum.

\section{Physical characterization of CNPs}

To verify the self-assembly of chimeric protein into nanoparticles in vitro, the purified CNPs was analyzed by TEM. Results showed under $\mathrm{pH} 8.0$ in $150 \mathrm{mM} \mathrm{NaCl}$ the purified chimeric 
235

236

237

238

239

240

241

242

243

244

245

246

247

248

249

250

251

252

253

254

255

256

257

258

259

260

261

262

263

264

proteins were assembled into nanoparticles with a diameter of 25-30nm (Fig.5A), which were consistent to the results of DLS (Fig.5B).

\section{Antibody induction in immunized mice}

To evaluate the immunogenicity of CNPs and TRE in vivo, anti-FMDV antibody titer in sera samples was evaluated by ELISA. As shown in Fig.6, the antibody titers were increased in a time-dependent way and specific antibodies against inactivated virus could be detected at 14 days post vaccination (dpv) in experimental group and PepVac group. On day $28 \mathrm{dpv}$ and $56 \mathrm{dpv}$, the CNPs group revealed the highest antibody levels, but no significant difference was found between CNPs group and PepVac group $(\mathrm{P}>0.05)$. Mice immunized with CNPs induced significantly higher antibody titer compared to TRE group $(\mathrm{P}<0.01)$ and PBS group $\mathrm{P}<0.0001)$. After booster immunization, antibody titers were further increased significantly except PBS group. Together, The CNPs displaying epitope 131-160 of FMDV showed the ability to induce a strong specific anti-FMDV humoral immune response compared to other groups.

\section{Tymphocyte proliferation}

The spleen lymphocytes were isolated from mice at 28 days after booster immunization and stimulated in vitro with inactivated FMDV. As shown in Fig.7, The specific lymphocyte response levels of CNPs, PepVac and TRE groups were significantly enhanced compared to PBS groups $(\mathrm{P}<0.01)$. The group of $\mathrm{CNPs}$ elicited higher lymphocyte proliferation responses than the TRE group $(\mathrm{P}<0.05)$, while no significant differences were observed between CNPs and PepVac groups $(\mathrm{P}>0.05)$.

\section{Cytokine assay}

As it has been well known that the ability of lymphocytes to secret cytokines after stimulation is positively correlated with their functions. Thus, it is necessary to detect the cytokine levels after the stimulation. To assess the cytokine secretion of spleen lymphocytes after stimulation, IFN- $\gamma$, IL-2 and IL-4 concentrations in culture supernatants were evaluated by ELISA. As shown in Fig.8, CNPs group and PepVac group induced greater IFN- $\gamma$ and IL-2 levels than PBS group (Fig.8A, B). However, there are no significant differences in the IL-4 level among three groups $(\mathrm{P}>0.05)$ (Fig.8C). Notably, the CNPs immunized group produced significantly higher IFN- $\gamma$ levels than TRE group and PepVac group $(p<0.05)$. Taken together, the data suggested that CNPs immunization could enhance T lymphocytes immune response by 
265

266

267

268

269

270

271

272

273

274

275

276

277

278

279

280

281

282

283

284

285

286

287

288

289

290

291

292

293

294

295

elevated proliferation rate and cytokines secretion.

\section{DISCUSSION}

FMDV remains as the main challenge in cloven-hoofed animals breeding worldwide (Sobrino et al. 2001), and innovative approach to produce safe and effective vaccine to prevent the occurrence and spread of FMDV is urgently needed. Infectious bursal disease subviral particles and porcine parvovirus subviral particles have been demonstrated to serve as an effective delivery and display platform for FMDV epitopes (Pan et al. 2016; Remond et al. 2009). However, these chimeric particles were obtained through eukaryotic cells, which limit the application of promotion in practice. Conveniently, the CP of MS2 phage could be expressed and assembled in E.coli, which makes it possible to achieve a rapid and low-cost production. Previous studies have shown that MS2 phage is a favorable platform for displaying and delivering epitope peptides (Fu \& Li 2016; Heal et al. 1999; Lino et al. 2017). However, the limited tolerance of MS2 phage to this insertion and the misfolded, aggregated or degraded proteins resulting from long amino acids insertion remained to be the main problems(Caldeira \& Peabody 2011; Peabody 1997). In this study, 30 amino acids polypeptide which came from the predominant epitope of FMDV were inserted into the coat protein of MS2 phage. Chimeric protein was expressed in E. coli, and can efficiently self-assembl into nanoparticles with a diameter of 25-30nm (Fig.5). Moreover, the epitope sequence 131-160 of VP1 was displayed on the surface of MS2, which was confirmed by DOT-ELISA (Fig.4). These results indicated that the insertion of 30 amino acids did not affect the folding and assembly of coat protein.

The FMDV VP1 G-H loop (141-160) is the main immunogenic epitopes for inducing neutralizing antibodies (Morgan \& Moore 1990; Ochoa et al. 2000). MS2 mediated VLPs vaccine displaying G-H loop (141-160) of FMDV provides 65\% protection against FMDV in guinea pigs and $60 \%$ protection against FMDV in pigs (Dong et al. 2015), which did not offer full protection. The flanking sequences of G-H loop can markedly strengthen its immune response (Dong et al. 2015; Fang et al. 2015). Moreover, the G-H loop of FMDV is an annular spatial conformation on the surface of natural virus particle, and correct conformation is crucial to induce effective humoral and cellular immune responses (Acharya et al. 1990;Cao et al. 2017; Rowlands et al. 1994). In the present research, we chose the epitope sequence 131-160 that contained G-H loop domain (141-160) and extensive flanking sequences to insert the AB loop of $\mathrm{CP}$ of MS2. And the epitope sequence 131-160 may still retain the circular structure through the 
296

297

298

299

300

301

302

303

304

305

306

307

308

309

310

311

312

313

314

315

316

317

318

319

320

321

322

323

324

325

326

327

AB loop of MS2. In fact, we had also attempted to insert epitope 124-167 of VP1 into the CP of MS2. But only a few chimeric proteins were obtained, which could not be assembled into nanoparticles (Shown in the supplemental information). This may be because insertions of 44 amino acids affected the CP of MS2 self-assembly. $15 \mu \mathrm{g}$ of CNPs stimulated enhanced humoral immune response than $30 \mu \mathrm{g}$ of TRE $(\mathrm{P}<0.01$, Fig.6), and induced slightly higher antibody levels compared to Pepvac, although there was no significant difference ( $\mathrm{P}>0.05$, Fig.6). These results indicated that the predominant epitope sequence 131-160 was displayed on the surface of MS2 at a high density, which stimulated stronger antibody levels in mice.

Based on G-H loop and C-terminal sequence (200-213) of VP1, a variety of epitope vaccines have been developed to elicit high neutralizing antibodies titers in small animals, such as mice and guinea pigs ( $\mathrm{Su}$ et al. 2007). However, several studies observed limited antibody levels and immune protection in host animals that induced by these epitope vaccines, which may be due to the lack of appropriate T-helper cell epitopes and low molecular weight of peptides (Cao et al. 2016; Rodriguez et al. 2003). CNPs, a special form of VLPs, had the capability of inducing extensive cell-mediated immune responses (Fu \& Li 2016; Ong et al. 2017). Notably, we observed elevated lymphocyte proliferation response in CNPs immunized mice than TRE group ( $\mathrm{P}<0.05$, Fig.7). Moreover, the secretion of IFN- $\gamma$ in CNPs group was significant enhanced compared to the TRE group and PepVac group ( $<<0.05$, Fig.8A, B) and the IL -2 levels in CNPs group was higher than the TRE group ( $<<0.05$, Fig.8B). It is well known that cytokine IFN- $\gamma$ and IL -2 are associated with cell-mediated immunity. Together with those results, we concluded that CNPs could elicit enhanced cell immune response compared to TRE and PepVac. Due to species differences, the following experiments will be performed to evaluate the immunogenicity and immuno-protection of CNPs in host animals.

In recent years, the purification processes of macromolecules, such as virus or VLPs, are mainly density gradient centrifugation and gel filtration chromatography. However, expensive and complicated purification processes limit the large-scale production of virus-like particles. (Dong et al. 2015; Liu et al. 2017; Pan et al. 2016). The new media Capto Core 700, with the capable of size separation and capture of small molecules, is designed for purification of viruses and other large biomolecules. In this study, we used gel filtration chromatography (Capto Core 700) for CNPs purification. Without flow rate limit and no repeated elution, the effluent containing CNPs could be collected directly. More importantly, the purity efficiency of CNPs and $\mathrm{CP}$ was over $85 \%$. 


\section{CONCLUSIONS}

329 In conclusion, we have developed a MS2 phage mediated CNPs, with displaying 330 predominant epitope 131-160 of VP1 on the CP of MS2 phage, which could be expressed and 331 self-assembled into nanoparticles in E.coli. In addition, the CNPs had improved immunogenicity 332 compared with PepVac and TRE, which could elicit enhanced specific anti-FMDV antibody 333 titers and elevated cellular immune response compared to TRE in mice. Therefore, MS2 phage334 mediated CNPs elicit an effective humoral and cellular immune responses in mice, which 335 provides a new perspective for future studies to subunit vaccine. 


\section{REFERENCES}

Acharya R, Fry E, Stuart D, Fox G, Rowlands D, and Brown F. 1990. The structure of foot-and-mouth disease virus: implications for its physical and biological properties. Vet Microbiol 23:21-34.

Burman A, Clark S, Abrescia NG, Fry EE, Stuart DI, and Jackson T. 2006. Specificity of the VP1 GH loop of Foot-andMouth Disease virus for alphav integrins. J Virol 80:9798-9810. 10.1128/jvi.00577-06

Caldeira JC, and Peabody DS. 2011. Thermal stability of RNA phage virus-like particles displaying foreign peptides. J Nanobiotechnology 9:22. 10.1186/1477-3155-9-22

Cao Y, Li D, Fu Y, Bai Q, Chen Y, Bai X, Jing Z, Sun P, Bao H, Li P, Zhang J, Ma X, Lu Z, and Liu Z. 2017. Rational design and efficacy of a multi-epitope recombinant protein vaccine against foot-and-mouth disease virus serotype A in pigs. Antiviral Res 141:133-141. 10.1016/j.antiviral.2017.01.023

Cao Y, Lu Z, and Liu Z. 2016. Foot-and-mouth disease vaccines: progress and problems. Expert Rev Vaccines 15:783789. 10.1586/14760584.2016.1141042

Chackerian B. 2007. Virus-like particles_flexible platforms for vaccine development.pdf. Expert Rev Vaccines 6:381390.

Chandramouli S, Medina-Selby A, Coit D, Schaefer M, Spencer T, Brito LA, Zhang P, Otten G, Mandl CW, Mason PW, Dormitzer PR, and Settembre EC. 2013. Generation of a parvovirus B19 vaccine candidate. Vaccine 31:3872-3878. 10.1016/j.vaccine.2013.06.062

Crisci E, Almanza H, Mena I, Cordoba L, Gomez-Casado E, Caston JR, Fraile L, Barcena J, and Montoya M. 2009. Chimeric calicivirus-like particles elicit protective anti-viral cytotoxic responses without adjuvant. Virology 387:303-312. 10.1016/j.virol.2009.02.045

Crisci E, Fraile L, Moreno N, Blanco E, Cabezon R, Costa C, Mussa T, Baratelli M, Martinez-Orellana P, Ganges L, Martinez J, Barcena J, and Montoya M. 2012. Chimeric calicivirus-like particles elicit specific immune responses in pigs. Vaccine 30:2427-2439. 10.1016/j.vaccine.2012.01.069

Diaz-San Segundo F, Medina GN, Stenfeldt C, Arzt J, and de Los Santos T. 2016. Foot-and-mouth disease vaccines. Vet Microbiol. 10.1016/j.vetmic.2016.12.018

Dong YM, Zhang GG, Huang XJ, Chen L, and Chen HT. 2015. Promising MS2 mediated virus-like particle vaccine against foot-and-mouth disease. Antiviral Res 117:39-43. 10.1016/j.antiviral.2015.01.005

Fang M, Wang H, Tang T, Zhao P, Du J, Guo S, Wei H, Xu H, Wan M, Wei X, Yu Y, and Wang L. 2015. Single immunization with a recombinant multiple-epitope protein induced protection against FMDV type Asia 1 in cattle. Int Immunopharmacol 28:960-966. 10.1016/j.intimp.2015.08.017

Fu Y, and Li J. 2016. A novel delivery platform based on Bacteriophage MS2 virus-like particles. Virus Res 211:9-16. 10.1016/j.virusres.2015.08.022

Heal KG, Hill HR, Stockley PG, Hollingdale MR, and Taylor-Robinson AW. 1999. Expression and immunogenicity of a liver stage malaria epitope presented as a foreign peptide on the surface of RNA-free MS2 bacteriophage capsids. Vaccine 18:251-258.

Koning RI, Gomez-Blanco J, Akopjana I, Vargas J, Kazaks A, Tars K, Carazo JM, and Koster AJ. 2016. Asymmetric cryo-EM reconstruction of phage MS2 reveals genome structure in situ. Nat Commun 7:12524. 10.1038/ncomms12524

Lino CA, Caldeira JC, and Peabody DS. 2017. Display of single-chain variable fragments on bacteriophage MS2 viruslike particles. J Nanobiotechnology 15:13. 10.1186/s12951-016-0240-7

Liu S, Yu X, Wang C, Wu J, Kong X, and Tu C. 2006. Quadruple antigenic epitope peptide producing immune 
protection against classical swine fever virus. Vaccine 24:7175-7180. 10.1016/j.vaccine.2006.06.042

Liu X, Fang Y, Zhou P, Lu Y, Zhang Q, Xiao S, Dong Z, Pan L, LV J, Zhang Z, Zhang Y, and Wang Y. 2017. Chimeric viruslike particles elicit protective immunity against serotype $O$ foot-and-mouth disease virus in guinea pigs. Appl Microbiol Biotechnol. 10.1007/s00253-017-8246-0

Ma L, Liu YS, Ding YZ, Chen HT, Zhou JH, Liu WQ, Wang M, and Zhang J. 2010. Preparation and characterization of neutralizing monoclonal antibodies against FMDV serotype $\mathrm{O}$ with synthetic peptide antigen. Hybridoma (Larchmt) 29:409-412. 10.1089/hyb.2010.0031

Mastico RA, Talbot SJ, and Stockley PG. 1993. Multiple presentation of foreign peptides on the surface of an RNAfree spherical bacteriophage capsid. J Gen Virol 74 ( Pt 4):541-548. 10.1099/0022-1317-74-4-541

Morgan DO, and Moore DM. 1990. Protection of cattle and swine against foot-and-mouth disease, using biosynthetic peptide vaccines. Am J Vet Res 51:40-45.

Ochoa WF, Kalko SG, Mateu MG, Gomes P, Andreu D, Domingo E, Fita I, and Verdaguer N. 2000. A multiply substituted G-H loop from foot-and-mouth disease virus in complex with a neutralizing antibody: a role for water molecules. J Gen Virol 81:1495-1505. 10.1099/0022-1317-81-6-1495

Ong HK, Tan WS, and Ho KL. 2017. Virus like particles as a platform for cancer vaccine development. PeerJ 5:e4053. $10.7717 /$ peerj.4053

Pan Q, Wang H, Ouyang W, Wang X, Bi Z, Xia X, Wang Y, and He K. 2016. Immunogenicity of adenovirus-derived porcine parvovirus-like particles displaying $\mathrm{B}$ and $\mathrm{T}$ cell epitopes of foot-and-mouth disease. Vaccine 34:578-585. 10.1016/j.vaccine.2015.11.003

Parida S. 2009. Vaccination against foot-and-mouth disease virus: strategies and effectiveness. Expert Rev Vaccines 8:347-365. 10.1586/14760584.8.3.347

Peabody DS. 1997. Subunit fusion confers tolerance to peptide insertions in a virus coat protein. Arch Biochem Biophys 347:85-92. 10.1006/abbi.1997.0312

Pereira HG. 1976. Subtyping of foot-and-mouth disease virus. Dev Biol Stand 35:167-174.

Remond M, Da Costa B, Riffault S, Parida S, Breard E, Lebreton F, Zientara S, and Delmas B. 2009. Infectious bursal disease subviral particles displaying the foot-and-mouth disease virus major antigenic site. Vaccine 27:9398. 10.1016/j.vaccine.2008.10.036

Rodriguez LL, Barrera J, Kramer E, Lubroth J, Brown F, and Golde WT. 2003. A synthetic peptide containing the consensus sequence of the G-H loop region of foot-and-mouth disease virus type-O VP1 and a promiscuous T-helper epitope induces peptide-specific antibodies but fails to protect cattle against viral challenge. Vaccine 21:3751-3756.

Rowlands D, Logan D, Abu-Ghazaleh R, Blakemore W, Curry S, Jackson T, King A, Lea S, Lewis R, Newman J, and et al. 1994. The structure of an immunodominant loop on foot and mouth disease virus, serotype 01, determined under reducing conditions. Arch Virol Suppl 9:51-58.

Sobrino F, Saiz M, Jimenez-Clavero MA, Nunez JI, Rosas MF, Baranowski E, and Ley V. 2001. Foot-and-mouth disease virus: a long known virus, but a current threat. Vet Res 32:1-30. 10.1051/vetres:2001106

Su C, Duan X, Wang X, Wang C, Cao R, Zhou B, and Chen P. 2007. Heterologous expression of FMDV immunodominant epitopes and HSP70 in P. pastoris and the subsequent immune response in mice. Vet Microbiol 124:256-263. 10.1016/j.vetmic.2007.04.030

Taboga O, Tami C, Carrillo E, Nunez JI, Rodriguez A, Saiz JC, Blanco E, Valero ML, Roig X, Camarero JA, Andreu D, Mateu MG, Giralt E, Domingo E, Sobrino F, and Palma EL. 1997. A large-scale evaluation of peptide 
418

419

420

421

422

423

424

425

426

427

428

429

430

431 vaccines against foot-and-mouth disease: lack of solid protection in cattle and isolation of escape mutants. J Virol 71:2606-2614.

Wang CY, Chang TY, Walfield AM, Ye J, Shen M, Chen SP, Li MC, Lin YL, Jong MH, Yang PC, Chyr N, Kramer E, and Brown F. 2002. Effective synthetic peptide vaccine for foot-and-mouth disease in swine. Vaccine 20:26032610.

Wei Y, Yang C, Wei B, Huang J, Wang L, Meng S, Zhang R, and Li J. 2008. RNase-resistant virus-like particles containing long chimeric RNA sequences produced by two-plasmid coexpression system. J Clin Microbiol 46:1734-1740. 10.1128/JCM.02248-07

Xu H, Bao X, Lu Y, Liu Y, Deng B, Wang Y, Xu Y, and Hou J. 2017. Immunogenicity of T7 bacteriophage nanoparticles displaying G-H loop of foot-and-mouth disease virus (FMDV). Vet Microbiol 205:46-52. 10.1016/j.vetmic.2017.04.023

Yang DF, Zhu HF, Wang ZH, Shen GX, and Tian DY. 2005. Construction of single chain Fv antibody against transferrin receptor and its protein fusion with alkaline phosphatase. World J Gastroenterol 11:3300-3303. 
Table $\mathbf{1}$ (on next page)

Sequences of primers for PCR/OE-PCR 
1 Sequences of primers for PCR/OE-PCR

\begin{tabular}{lr}
\hline Primer name & Primer sequence $\left(5^{\prime}-3^{\prime}\right)$ \\
\hline MS2-F & CGggatccGTGCGAGCTTTTAGTACCCTTGA \\
MS2-R & CCCaagcttTGTTGTCTTCGACATGGGTAATCCTC \\
IN-F & \\
CTGACCAACGTGCGTGGCGATCTGCAAGTCCTGGCACAGAAAGCTGCACGTCCTC \\
TGCCTACTGGCGACGTGACTGTCGCCCCAAGCAA \\
IN-R \\
TGCCAGGACTTGCAGATCGCCACGCACGTTGGTCAGGGAACCCTCAGCGTACTTG \\
\hline CAGTTCCCGTTTCCGCCATTGTCGACGAGAACGAAC
\end{tabular}

2 Notes

3 Lowercase part in primers MS2-F and MS2-R represent for restriction enzymes (BamH I and Hind III).

4 The sequences in bold type in two primers IN-F and IN-R were the epitope sequence 131-160 of VP1, and underlined 36

5 nucleotides were reverse complementary. 


\section{Figure 1}

Schematic drawing of constructing process for recombinant plasmids.

( $A$ and $C$ ) Schematic diagram of recombinant plasmid $\mathrm{pCP}$ and $\mathrm{p}-\left(\mathrm{EP}_{131-160}\right)_{3}$. (B) Strategy to construct recombinant plasmid $\mathrm{pCP}-\mathrm{EP}_{131-160}$.

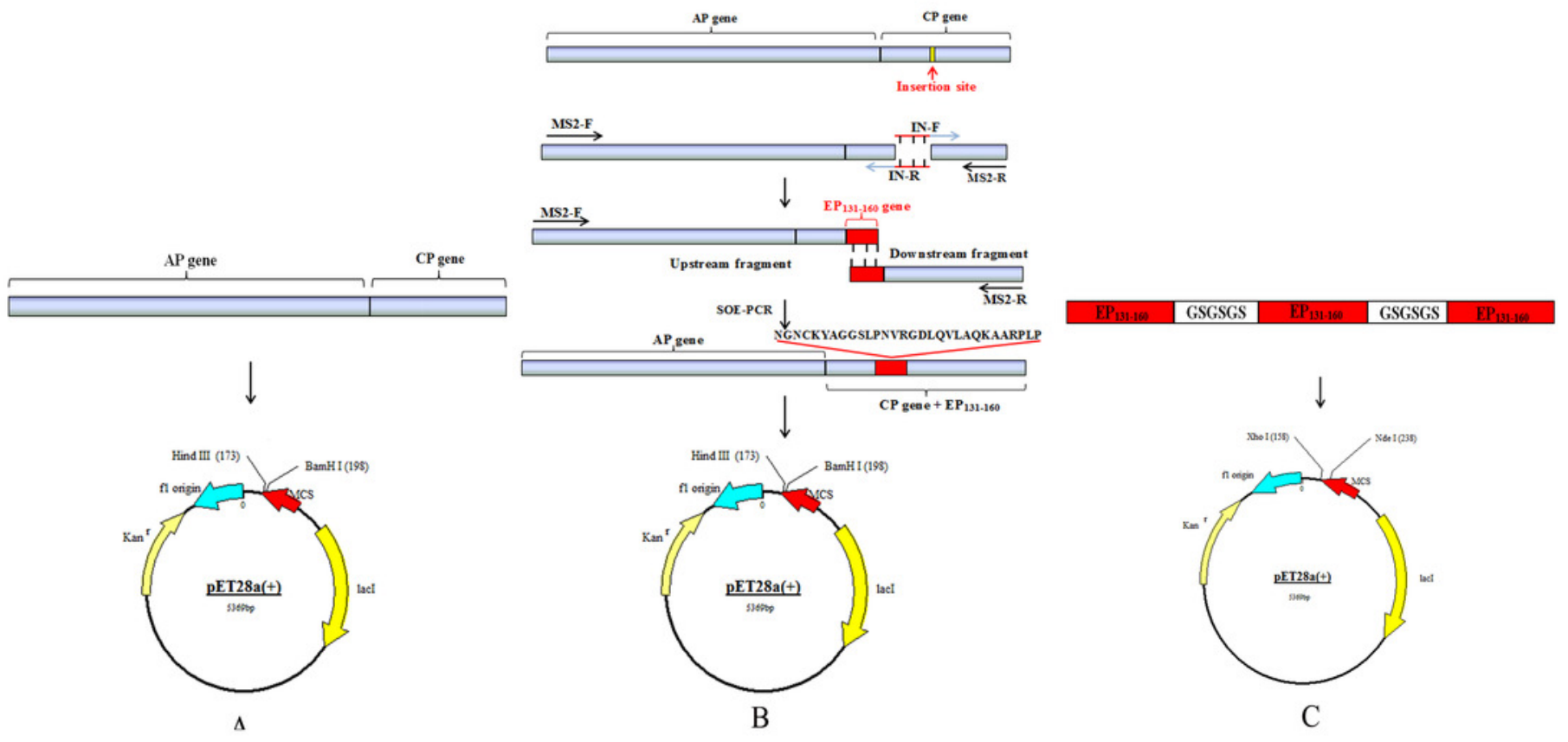




\section{Figure 2}

Amplification of target gene and identification of recombinant plasmids.

(A) PCR amplification products analysis using agarose gel. Lane 1, DL2000 DNA ladder; Lane 2, Mature protein (AP) and coat protein (CP) genes of MS2 showing 1644 bp amplicon; Lane 3, Upstream fragment showing 1260 bp amplicon; Lane 4, Downstream fragment showing 487 bp amplicon; Lane 5, The whole fragment ( $\left.A P+C P+E_{131-160}\right)$ showing 1734 bp amplicon. (B) The identification of recombinant plasmids by digestion with restriction enzymes. Lane 1, DL2000 DNA ladder; Lane 2-3, Recombinant plasmids PCP and PCP-EP ${ }_{131-160}$ were digested using restriction enzymes BamH I and Hind III, respectively; Lane 4, Recombinant plasmid p$\left(\mathrm{EP}_{131-160}\right)_{3}$ was digested by $\mathrm{Nde} \mathrm{I}$ and $\mathrm{Xol} \mathrm{I}$.

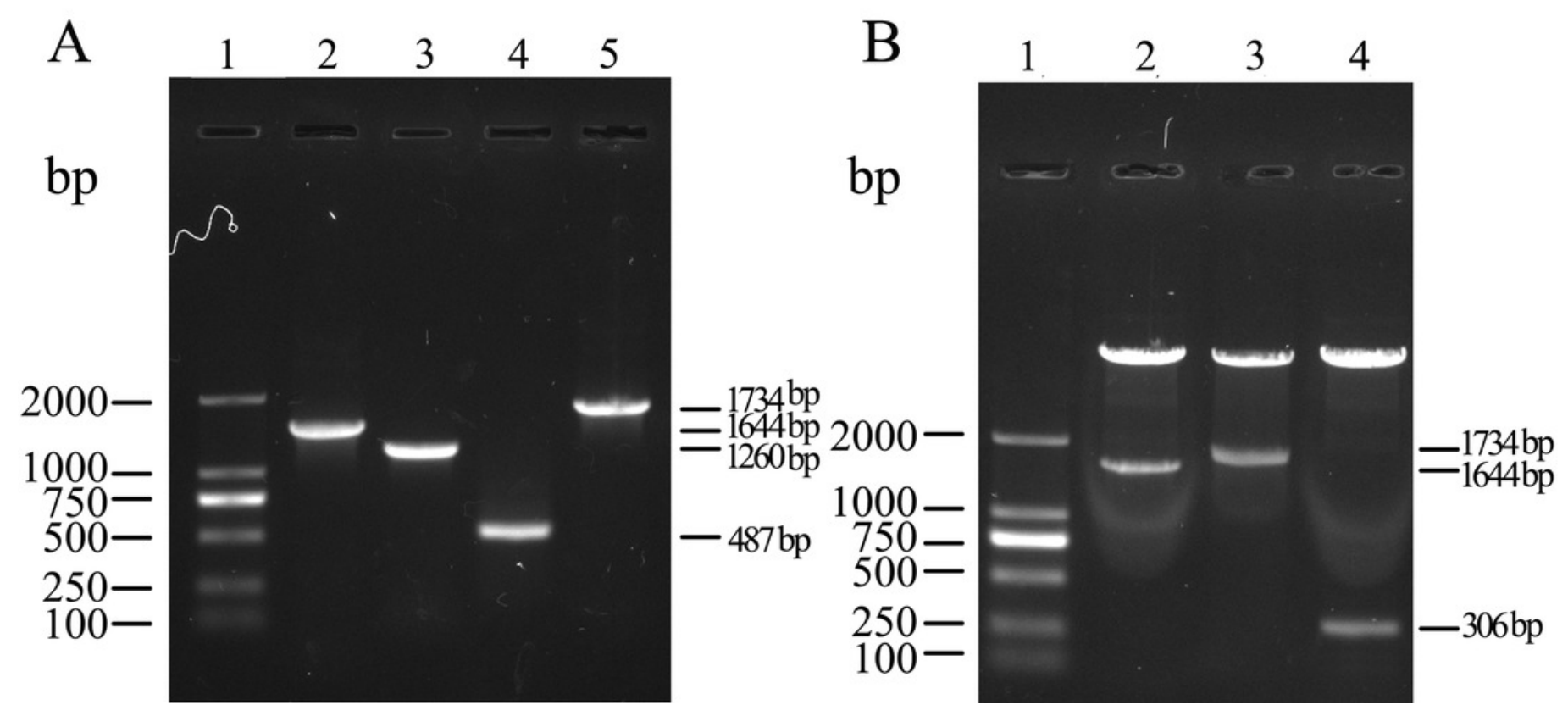




\section{Figure 3}

Expression and purification of recombinant proteins.

(A) SDS-PAGE analysis of the solubility of three recombinant proteins. Lane 1, Protein ladder; Lane 2-3, Level of coat protein of MS2 expressed in soluble and insoluble fractions, respectively; Lane 4-5, Level of chimeric protein expressed in soluble and insoluble fractions, respectively; Lane 6-7, Level of tandem repeat peptide epitopes (TRE) expressed in soluble and insoluble fractions, respectively. As shown, coat protein of MS2, chimeric protein and TRE were detected with a molecule weight near $16 \mathrm{kDa}, 18 \mathrm{kDa}$ and $10 \mathrm{kDa}$, respectively. (B) Western-blot analysis of recombinant proteins with anti-FMDV VP1 G-H loop monoclonal antibody. Lane 1-7, The order is consistent with SDS-PAGE (A). (C) SDS-PAGE analysis of the purity of three recombinant proteins. Lane 1, Protein ladder; Lane 2, Purified coat protein of MS2; Lane 3, Purified chimeric protein; Lane 4, Purified TRE.

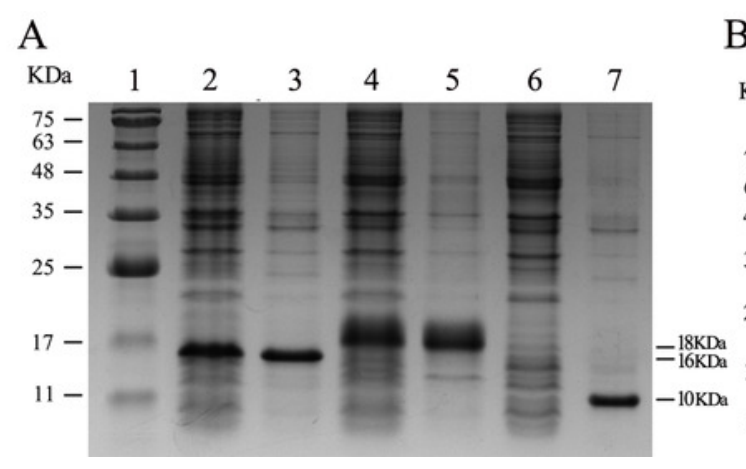

B

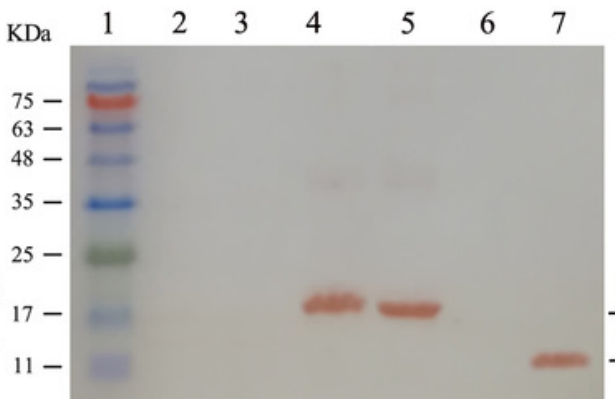

C

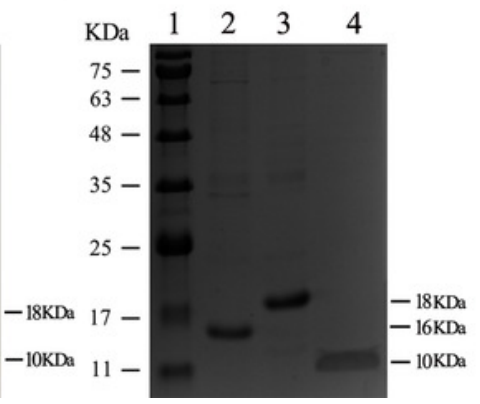


Figure 4

Immune-activity analysis of recombinant proteins.

Dot-ELISA immune assay with guinea pig anti-FMDV hyper-immune serum. (B) Dot-ELISA immune assay with anti-FMDV VP1 G-H loop monoclonal antibody. 1, Inactivated FMDV; 2, Purified coat protein of MS2; 3, Purified Chimeric nanoparticles; 4, Purified TRE.
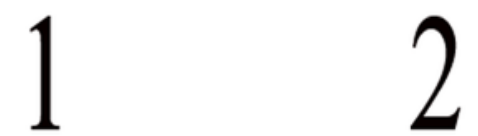

3

4

A

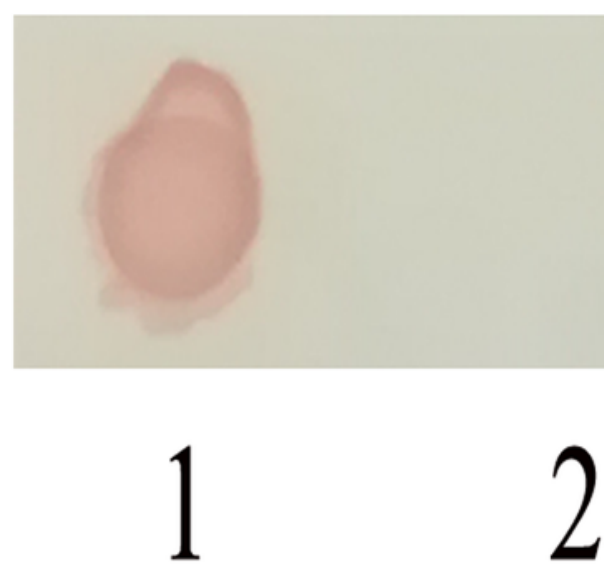

2

3

4 


\section{Figure 5}

Physical characterization of chimeric nanoparticles (CNPs).

(A) Transmission electron microscope (TEM) image of negative staining CNPs. The scale is $100 \mathrm{~nm}$. (B) Dynamic light scattering results of CNPs.

A

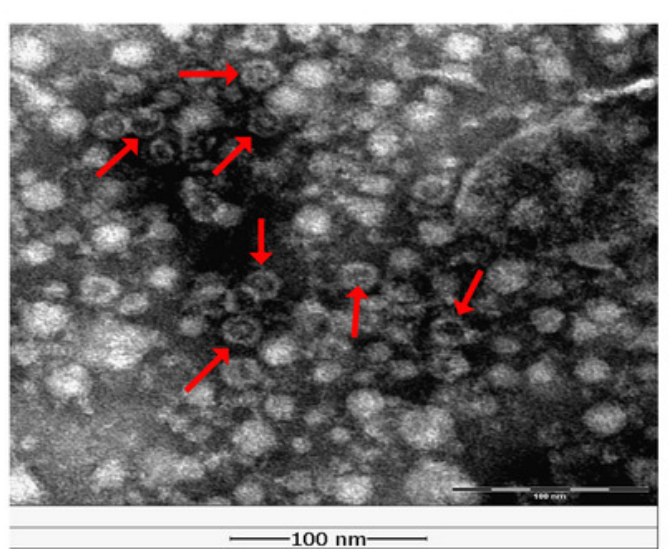

B

Size Distribution by Number

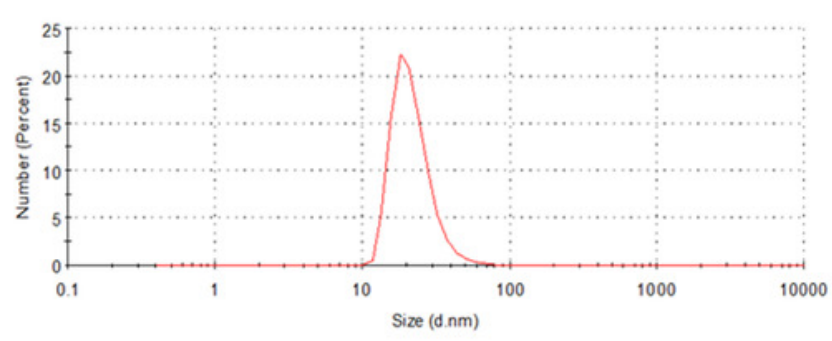

Record 36: 3 


\section{Figure 6}

CNPs specific antibody levels in mice.

Groups of mice were immunized with $15 \mu \mathrm{g}$ chimeric nanoparticles, $30 \mu \mathrm{g}$ tandem repeat peptide epitopes, commercialized synthetic peptide vaccine (more than $7.5 \mu \mathrm{g}$ ) and PBS.

Sera were collected at every week after the first immunization for antibody levels until the eighth week. Date are presented as the mean \pm SEM. Statistical differences were indicated by asterisk $\left({ }^{*} \mathrm{P}<0.05,{ }^{* * * *} \mathrm{P}<0.0001\right)$.

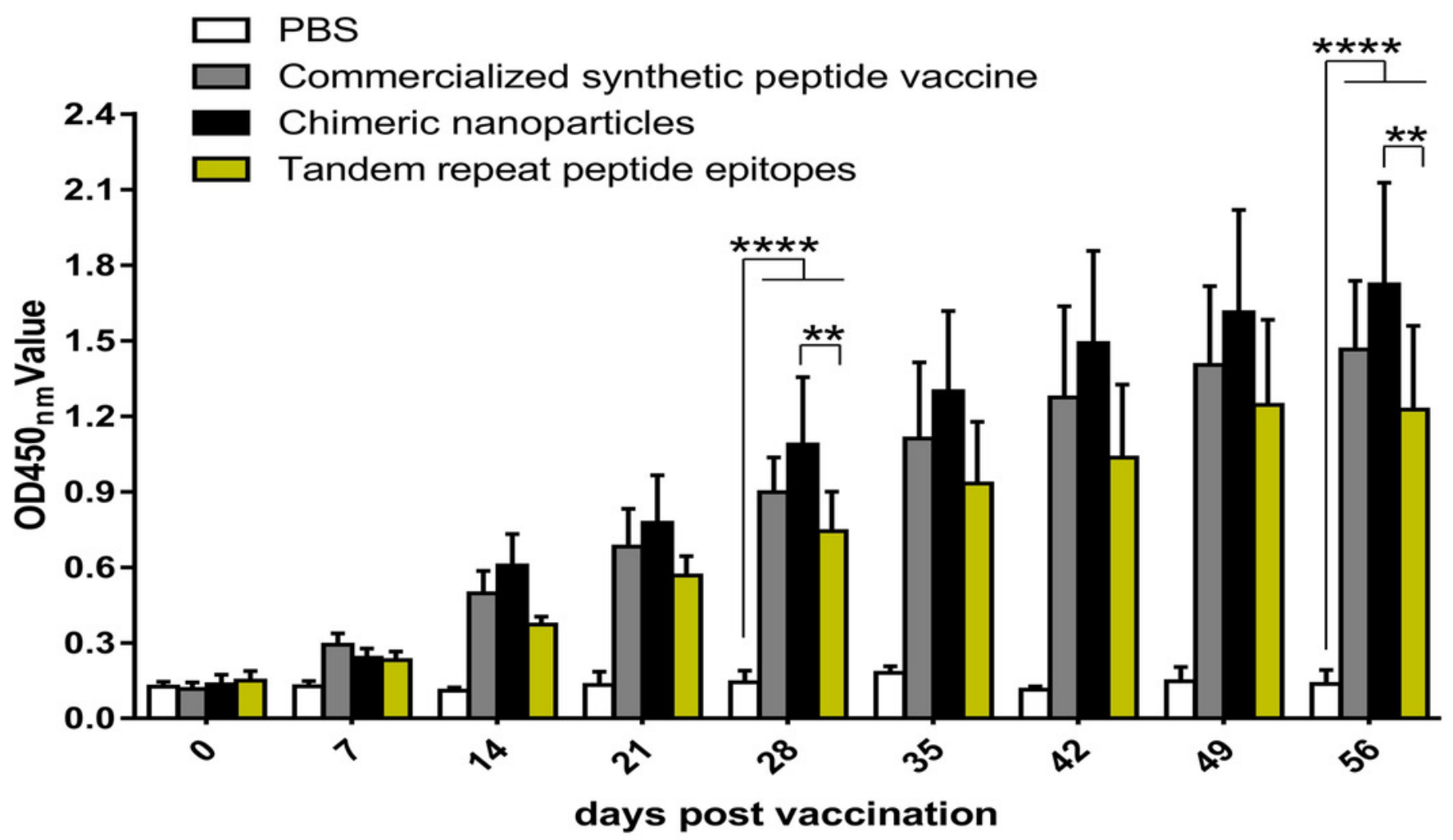


Figure 7

T-lymphocyte proliferation in mice.

Splenic Lymphocytes were isolated at 56 days post vaccination and stimulated with inactivated FMDV and concanavalin (CoA), respectively. Lymphocyte proliferation was analyzed using the WST-8 colorimetric assay. The stimulation index (SI) means the ratio of stimulated sample to unstimulated sample at $\mathrm{OD}_{450} \mathrm{~nm}$. Statistical differences were indicated by asterisk $\left({ }^{*} \mathrm{P}<0.05,{ }^{*} \mathrm{P}<0.01,{ }^{*} * \mathrm{P}<0.001\right)$.

\section{$\square$ Concanavalin A $\square$ Inactivated vaccine}

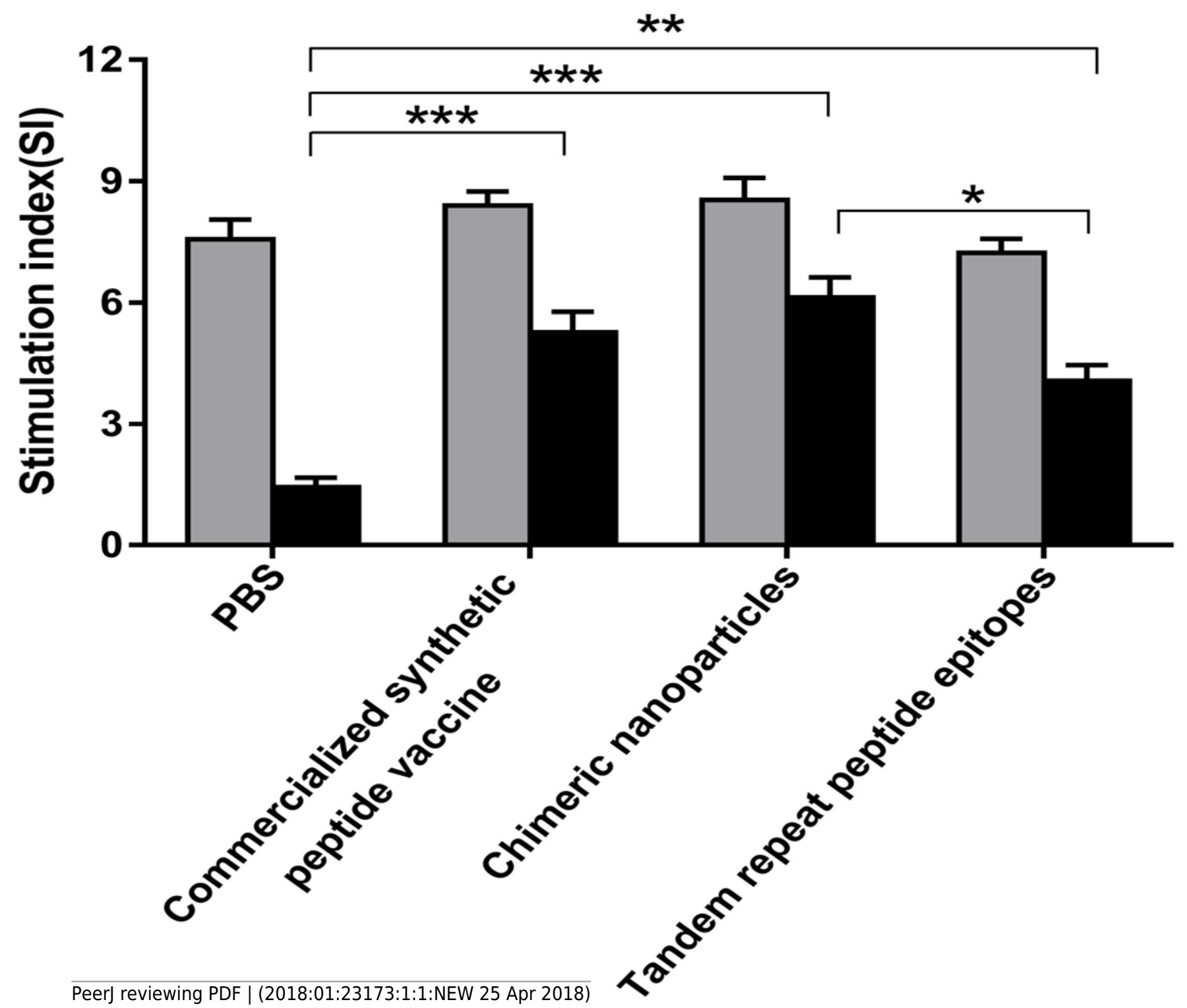


Figure 8

Analysis of cytokines secreted by lymphocyte of mice.

Splenic Lymphocytes were stimulated with inactivated FMDV for $48 \mathrm{~h}$. The culture supernatants were collected and tested by ELISA. (A), (B) and (C) are the concentrations $(\mathrm{pg} / \mathrm{mL})$ of IFN- $\gamma, \mathrm{IL}-2$ and IL-4 in the supernatants, respectively. Data are shown as mean \pm SEM. Statistical differences were indicated by asterisk $\left({ }^{*} \mathrm{P}<0.05,{ }^{*} \mathrm{P}<0.01\right.$, $\left.{ }^{* * *} \mathrm{P}<0.001\right)$.

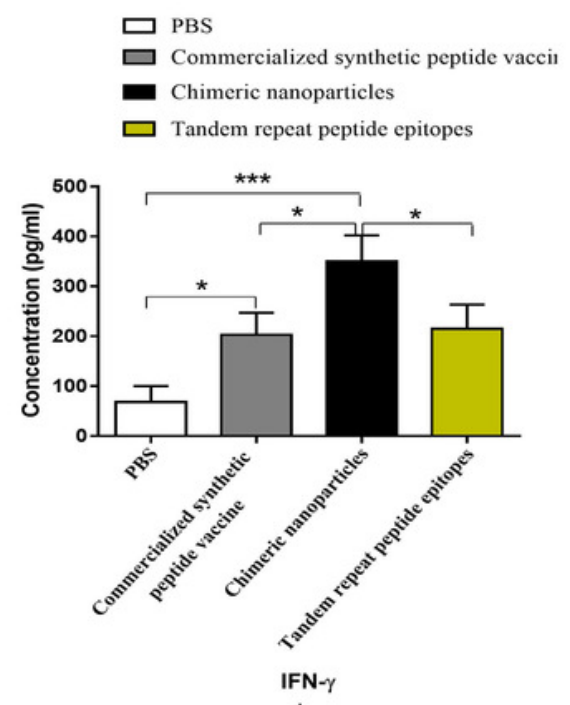

A

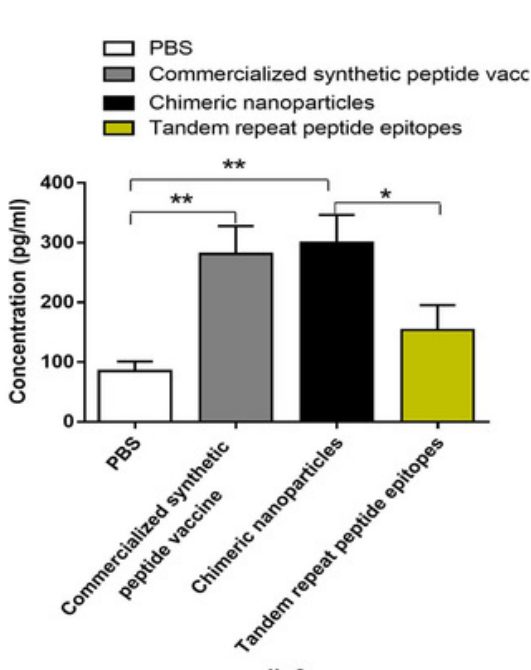

B.:-2

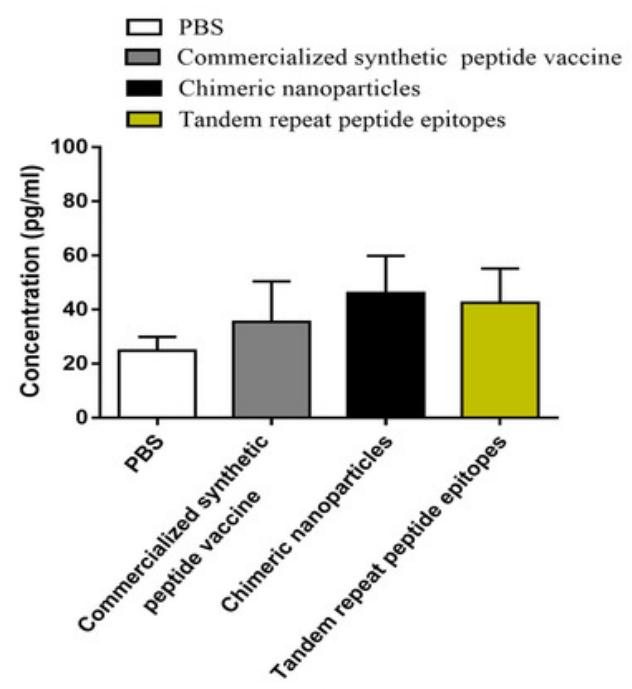

" 